\title{
TEMPLATE-FREE RIBOSOMAL SYNTHESIS OF POLYPEPTIDES \\ FROM AMINOACYL-tRNAS : POLYPHENYLALANINE \\ SYNTHESIS FROM PHENYLALANYL-tRNALYs
}

\section{G. Zh. Yusupova (Tnalina), N. V. Belitsina, A. S. Spirin}

Institute of Protein Research,

Academy of Sciences of the USSR, Pushchino ${ }_{n}$ Moscow Region

A. N. Bach Institute of Biochemistry, Academy of Sciences of the USSR, Moscow

\section{$\mathrm{Sum} \mathrm{m}$ a r y}

Misacylated phenylalanyl-tRNA ${ }^{\text {Lys }}$, similar to lysyl-tRNA ${ }^{\mathrm{Lys}}$, but not phenylalanyltRNA $^{\text {Phe }}$, is able to serve as a substrate for ribosomal synthesis of polypeptides (polyphenylalanine and polylysine, respectively) in the absence of a template polynucleotide (poly $(A))$. Thus, it is the structure of tRNA that determines the ability of the aminoacyl-tRNA ${ }^{\text {Lys }}$ to participate in the peptide elongation on ribosomes without codon-anticodon interactions.

1. Belitsina N. V., Tnalina G. Zh., Spirin A. S. Template-free ribosomal synthesis of polylysine from lysyl-tRNA// FEBS Letters.-1981.-131, N 2.-P. 289-292.

2. Belitsina $N . V$. Tnalina $G . Z h$. Spirin $A . S$. Template-free ribosomal synthesis of polypeptides from aminoacyl-tRNAs // Biosystems. - 1982.-15, N 3.-- P. 233-241.

3. Тналина $\Gamma$. Ж., Белицина H. B., Спирин A. C. Безматричный синтез полипептидов из аминоацил-тРНК на рибосомах Escherichia coli // Докл. АН CCСР.- 1982,-266, № 3.- C. 741-745.

4. Pestka $S$. Studies on the formation of transfer ribonucleic acid-ribosome complexes. III. The formation of peptide bonds by ribosomes in the absence of supernatant enzymes // J. Biol. Chem.-1968.-243, N 10.-P. 2810-2820.

5. Erbe R. W., Nau M. M., Leder P. Translation and translocation of defined RNA messenger // J. Mol. Biol.-1969.-39, N 3.-P. 441-460.

6. Kaziro K. Ynoue-Yokosawa N. Kawakita $M$. Studies on polypeptide elongation factor from E. coli. I. Crystalline factor G.// J. Biochem.-1972.-7.2, N 4.- P. 853-863.

7. Arai K., Kawakita M., Kaziro Y. Studies on polypeptide elongation factors from Escherichia coli. II. Purification of factors $T_{u}$-guanosine diphosphate, $T_{s}$ and $T_{u}-T_{s}$, and crystalization of $T_{u}$-guanosine diphosphate and $T_{u}-T_{s} / / \mathrm{J}$. Biol. Chem.-1972.247, N 21.- P. 7029-7037.

8. Fischer W., Derwenskus $K .-H$., Sprinzl $M$. On the properties of immobilized elongation factor $T_{u}$ from Thermus thermophilus HB8//Eur. J. Biochem,-1982.-125, N 1.P. $143-149$.

9. Гаврилова Л. П., Смолянинов В. В. Изучение механизма транслокации в рибосомах. I. Синтез полифенилаланина в рибосомах Escherichia coli без участия гуанозин-5'трифосфата и белковых факторов трансляции / Молекуляр. биология.-1971.-5, № 6.-C. $883-891$.

10. Wagner $f$., Sprinzl $M$. The complex formation between Escherichia coli aminoacyltRNA, elongation factor $T_{u}$ and GTP // Eur. J. Biochem.- 1980.-108, N 1.-P. 213221

11. Synthetic polynucleotides and the amino acid code, VIII / R. S. Gardner, R. S. Wahba, A. C. Basilioc et al. // Proc. Nat. Acad. Sci. USA.-1962.-48, N 12.- P. 2087-2094.

12. Gottesman M. E. Reaction of ribosome-bound peptidyl transfer ribonucleic acid with aminoacyl transfer ribonucleic acid or puromycin //J. Biol. Chem.-1967.-242, N 23.- P. 5564-5571.

Ин-т белка АН СССР, Пущино

Нн-т биохимии им. А. Н̈. Баха АН СССР, Москва

Получено 10.02 .86

УдК 547.963 .3

\section{СТРУКТУРА КАЛЬЦИЕВОЙ СОЛИ РОLY (dA) : POLY (dT) ПО ДАННЫМ РЕНТГЕНОВСКОЙ ДИФРАКЦИИ В ВОЛОКНАХ}

\section{Д. Г. Алексеев, А. А. Липанов, И. Я. Скуратовский}

Введение. Метод рентгеновской дифракции в волокнах оказался весьма успешным при изучении взаимодействия полимерной ДНК с противоионами и позволил локализовать ионы $\mathrm{Cs}^{+}$в структурах В- и A-форм

\footnotetext{
* Представлена членом редколлегии д. ф.-м. н. М. Д. Франк-Каменецким.
} 
ДНК $[1,2]$. Ионы щелочных металлов достаточно большого размера $\left(\mathrm{Na}^{+}, \mathrm{K}^{+}, \mathrm{Rb}^{+}\right)$могут располагаться в структуре ДНК сходным образом. В частности, оказывается возможным встраивание ионов $\mathrm{Na}^{+}$в гликозидный желобок poly $(\mathrm{dA})$ : poly $(\mathrm{dT})$ в классической B-конформации так, что в их координационные оболочки входят молекулы воды и полярные атомы оснований: N3 аденинов и О2 тиминов [1].

Двухвалентный ион $\mathrm{Mg}^{2+}$ обладает очень малым радиусом, его гидратационные свойства заметно отличаются от свойств упомянутых ионов, и о механизме его влияния на структуру ДНК можно судить лишь косвенно $[1,3] . \mathrm{Ca}^{2+}$ имеет такой же размер, как и $\mathrm{Na}^{+}$, но по данным EXAFS-спектроскопии его взаимодействие $c$ природной ДНК и poly $(\mathrm{dA})$ : poly $(\mathrm{dT})$ решительно отличается от того, что характерно для ионов щелочных металлов с примерно таким же размером: ионы $\mathrm{Ca}^{2+}$ взаимодействуют преимущественно с фосфатами и не имеют контактов с атомами азотистых оснований [4].

Настоящая работа посвящена детальной структуре poly $(\mathrm{dA})$ : poly $(\mathrm{dT})$ в форме кальциевой соли в волокнах и позволит более конкретно описать взаимодействие двухвалентного иона $\mathrm{Ca}^{2+} \mathrm{c}$ полимерной ДНК. В результате рентгеноструктурного исследования натриевой соли poly $(\mathrm{dA})$ : poly (dT) Арнотт и др. получили для этого полинуклеотида гетерономную структуру [5] с явно выраженной неэквивалентностью конформаций полипуриновой и полипиримидиновой цегей, что, в принципе, согласуется с их химической неэквивалентностью (цепь poly (dA) в данной модели принимает конформацию А-типа с сахаром ' $3^{\prime}$-endo, а цепь poly $(\mathrm{dT})$ - конформацию В-типа с сахаром $\mathrm{C} 2^{\prime}$-endo). Данные спектроскопических исследований в растворе (ЯМР, комбинационное рассеяние [6--8]), выполненных в различных условиях, не всегда согласуются с этой структурой. Қак будет показано ниже, в случае Ca-poly $(\mathrm{dA})$ : poly $(\mathrm{dT})$ химическая неэквивалентность двух цепей полинуклеотида практически не проявляется вовсе, а для Na-poly $(\mathrm{dA})$ : : poly (dT) в волокнах удается построить модель, где эта неэквивалентность выражена существенно слабее, чем в прежней модели структуры [5], причем новая модель оказывается в лучшем соответствии с теми же рентгеноструктурными данными.

Материалы и методы. Натриевую coль poly $(\mathrm{dA})$ : poly $(\mathrm{dT})$ («Böehringer», ФРГ) растворяли в буфере $0,1 \times \mathrm{SSC}$ и диализовали несколько раз против $1 \mathrm{mM}$ трис-НCl, $\mathrm{pH} 7,8$, содержащего $10 \mathrm{mM} \mathrm{CaCl}$. Осажденные после диализа препараты тщательно отмывали $80 \%$-ным этанолом, в результате чего получали чистую кальциевую соль роly $(\mathrm{dA}):$ poly $(\mathrm{dT})$, свободную от примеси $\mathrm{CaCl}_{2}$. На CuKa-излучении была снята рентrенограмма Ca-poly (dA): poly(dT) при относительной влажности $81 \%$ и измерены интенсивности 87 независимых огражений. В отличие от Na-poly $(d \mathrm{~A})$ : poly $(\mathrm{dT})$, когда при относительной влажности $71 \%$ наблюдали моноклинную элементарную ячейку с параметрами: $a=1,865 \pm 0,002$ нм, $b=3,548 \pm 0,002$ нм, $c=3,233 \pm 0,003$ нм, $\beta \approx 90^{\circ}$ (пространствснная групла $\mathrm{P} 2_{1}$ ) [5], в случаe Ca-poly (dA) : poly (dT) наблюдается ромбическая ячейка с параметрами: $a=1,871 \pm 0,001 \mathrm{нм}, b=4,032 \pm 0,002 \mathrm{Hм}, c=3,232 \pm$ $+0,003$ нм (пространственная группа $\mathrm{P} 2_{1} 2_{1} 2_{1}$ ). Таким образом, при переходе от $\mathrm{Na}$ к Ca-poly (dA) : poly (dT) происходит увеличение длины ребра «b» элементарной ячейки на 0,48 нм при совпадении остальных параметров. В обонх случаях молекулы роly $(\mathrm{dA})$ : poly (dT) представляют собой 10-кратные двойные спирали с одинаковой высотой витка 3,23 нм, и на ячейку приходится две молекулы. Анализ систематических погасаний и цилиндрически усредненной функции Паттерсона (получаемой из экспериментальных интенсивностей без каких-либо априорных допущений, см., например, [9]) свидетельствует, что две молекулы Ca-poly(dA) : poly $(\mathrm{dT})$ в ячейке связаны друг с другом трансляцией, и их положения в долях ребер ячсйки следующие: $(0 ; 0 ; 0)$ и $(0,5 ; 0,5 ; 0,31)$.

Оптимизацию структуры полинуклеотида проводили с помощью модифицированного метода связанного атома Арнотта и др. [10]. Использовали однопараметрическую модель гибкого сахарного кольца [11], рассчитанную по статистике известных структур нуклеотидов и нуклеозидов из кембриджского банка кристаллографических данных (версия 1984 г.). Оптимизированные структуры считали стерически приемлемыми, если они не содержали контактов между валентно несвязанными атомами (в том числе и атомами водорода) короче, чем сумма их ван-дер-ваальсовых радиусов минус 0,04 нм. Рассеивающие факторы нсводородных атомов были поправлены на дифракцию от неупорядочснного растворителя (воды) в соответствии с [12], однако при всех расчстах не учитывали «малоугловые» рефлексы (до разрешения 1,1 нм), где өтот вклад наиболее существен. В случае Ca-poly (dA) : poly(dT) иместся всего три таких рефлекса из 87 , а в случаe Na-poly (dA) : poly (dT) - семь из 113. 
Результаты. Структур а C a-poly $(\mathrm{d} A)$ : poly (d T). Анализ синтезов Паттерсона позволил пірямо показать наличие в структуре Ca-poly (dA) : poly (dT) традиционного набора некристаллографических диад (поворотных осей второго порядка), перпендикулярных оси спирали. Хотя такие диады не являются абсолютно строгими, так как адениновые и тиминовые основания в противоположных цепях двойной спирали только квазиизоморфны, этот факт исключает возможность заметной неэквивалентности цепей poly $(\mathrm{dA}): \operatorname{poly}(\mathrm{d} \mathrm{T})$, т. е. позволяет

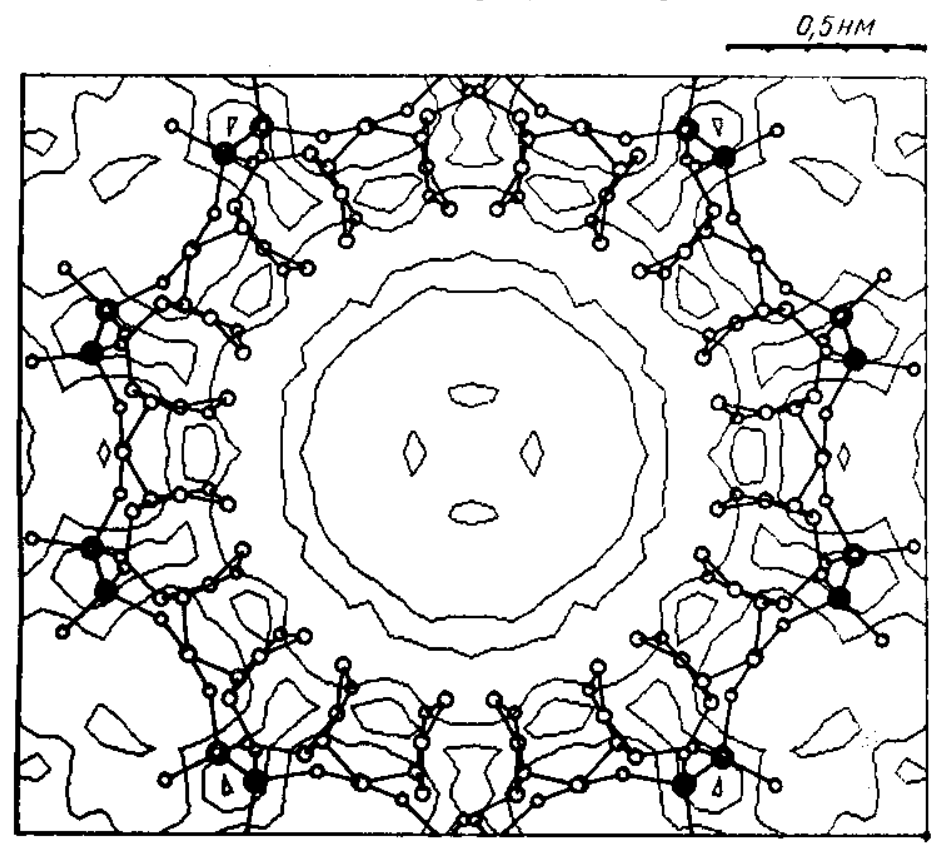

Рис. 1. Квадрат электронной плотности молекулы Ca-poly $(\mathrm{dA}):$ poly(dT) за вычстом константы, рассчитанный по трехмсрной функции Паттерсона. Проекция вдоль оси спнрали, которая перпендикулярна плоскости рисунка. Положение максимумов электронной плотности хорошо согласуется с наложенным изображением сахаро-фосфатных цепей уточненной модели. Атомы фосфора зачернены и их положение соответствует расстоянию 0,96 нм от оси спирали (центр рнсунка).

Fig. 1. The squared electron density of the Ca-poly $(\mathrm{dA})$ : poly(dT) molecule (minus a constant value) calculated from the three-dimensional Patterson map. Projection down the molecular screw axis which is normal to the plane of the picture. The positions of electron density maxima coincide well with those of phosphates from the refined model of the structure. Black circles indicate the phosphorous atoms $0.96 \mathrm{~nm}$ distant from the helix axis (centre of the picture).

сразу отвергнуть гетерономную модель, предложенную для натриевой соли этого полинуклеотида [5]. Зная пространственную группу, положение внутримолекулярных диад и учитывая 10-кратную винтовую - симметрию отдельных молекул Ca-poly $(\mathrm{dA})$ : poly $(\mathrm{dT})$, можно, воспользовавшись трехмерным синтезом Паттерсона, рассчитать квадрат электронной плотности молекулы и найти положение фосфатных групл в ячейке. На рис. 1 показана проекция полученного изображения молекулы вдоль оси спирали. Важно, что этот результат следует непосредственно из экспериментальных данных и свободен от неопределенностей, которые могут быть присущи (в принципе) поискам структуры с помощью моделирования.

Относительно независимое определение структуры молекулы методом связанного атома согласуется с результатами анализа функции Паттерсона. Обычный $R$-фактор $\left(R=\Sigma\left|F_{\ni}-F_{T}\right| / \Sigma F_{9}\right.$, где $F_{\vartheta}-$ экспериментальные, а $F_{T}$ - теоретические структурные амплитуды дифракции) для окончательной структуры равен 0,32. Используемый для статистических оценок $R^{\prime \prime}\left(R^{\prime \prime}=\left(\Sigma\left(F_{\ni}-F_{T}\right)^{2} / \Sigma F_{y^{2}}\right)^{1 / 2}\right)$ равен 0,36 . Оптимизированная структура характеризуется одинаковыми параметрами противоположных цепей двойной спирали с сахарами в конформации, близкой 
к C2'-endo. Пары оснований имеют отрицательный наклон по отношению к плоскости, перпендикулярной этой оси $\left(-6^{\circ}\right)$, и между аденином и тимином в каждой из пар существует значительный пропеллер $\left(19^{\circ}\right)$. В целом структура принадлежит, несомненно, к В-семейству ДНК.

Cтруктура N a-poly(d A): poly(d T). Ввиду близких значений упаковочных и молекулярных параметров для кальциевой и натриевой солей poly $(\mathrm{dA})$ : poly (dT) (см. Материалы и методы) мы попы-
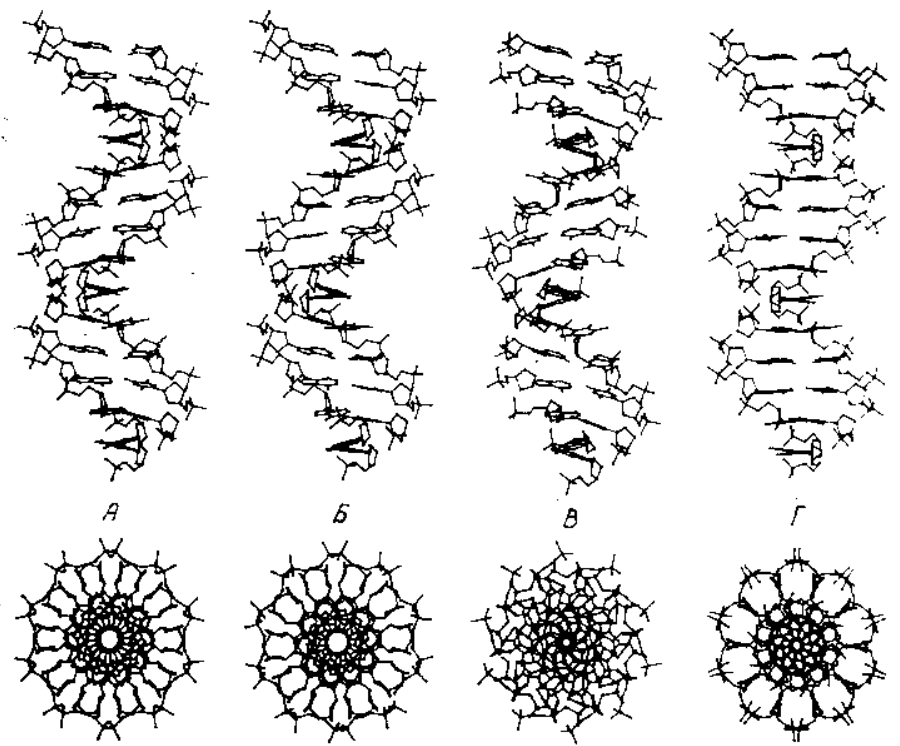

Рис. 2. Проекции перпендикулярно (вверху) и вдоль (внизу) оси спирали кальциевой $(A)$, натриевой $($ E) солей poly $(\mathrm{dA})$ : poly $(\mathrm{dT})$; гетерономной модели $\mathrm{Na}$-poly $(\mathrm{dA})$ : ро$\operatorname{ly}(d \mathrm{~T})(B)[5]$ и модели В-ДНК $(T)[5]$.

Fig. 2. Views normal to (above) and down (below) the helix axis of $A$ ) Ca-poly $(\mathrm{dA})$ : : poly $(\mathrm{dT})$; 5$) \mathrm{Na}$-poly (dA) : poly (dT) (this work); $B$ ) heteronomous model of Na-poly (dA) : poly (dT) [5]; $\Gamma$ ) B-DNA model [5].

тались адаптировать полученную модель к данным рентгеновской дифракции от волокон Na-poly $(\mathrm{dA})$ : poly $(\mathrm{dT})$ из работы [5]. B результате оптимизации была найдена структура, у которой конформации сахаров в противоположных цепях отличаются, но обе близки к $\mathrm{C}^{\prime}$ endo. При этом различия в двугранных углах между адениновой и тиминовой цепями в целом невелики (стандартное отклонение $13^{\circ}$ ), тогда как в гетерономной модели [5] эти различия составляют $45^{\circ}$. Оптимизированная структура осталась очень похожей на структуру Ca-poly $(\mathrm{dA})$ : poly $(\mathrm{dT})$. Для нашей модели Na-poly $(\mathrm{dA})$ : poly $(\mathrm{dT})$ $R=0,26\left(R^{\prime \prime}=0,29\right)$. Гетерономная модель в тех же условия х дает $R$-фактор $0,28 \quad\left(R^{\prime \prime}=0,37\right)$, и, согласно статистическому тесту Гамильтона [13], может быть отвергнута при уровне достоверности 99,5\%.

С равнение разлияных структур poly(dA):poly(dT). $\mathrm{Ha}$ pис. 2 изображены полученные нами модели $\mathrm{Ca}-$ и $\mathrm{Na}$-poly (dA) : : poly(dT), а также гетерономная модель [5] и модель В-ДНК. На рис. 3 показаны виды сверху на две последовательные пары нуклеотидов из этих структур, а в таблице приведены значения соответствующих им конформационных параметров, Все три модели poly (dA) : : poly (dT) характеризуются сходным стэкингом между парами оснований, несмотря на различия в конформациях сахаро-фосфатных цепей (рис. 3), и значительной величиной пропеллера между основаниями в каждой из пар. Существенно то, что наша модель Na-poly (dA) : : poly (dT) (рис. $2, \bar{b}$ ) требует иного взаимного расположения молекул в элементарной ячейке (при сохранении ее экспериментальных размеров и пространственной группы $\mathrm{P} 2_{1}$ ), чем модель [5] (pис. 2, $B$ ). 
Обсуждение результатов. Приведенные результаты и данные ЯМР [6] показывают, что в волокнах и при физиологических условня х в растворе (температура $\sim 25^{\circ} \mathrm{C}, 0,1 \mathrm{M} \mathrm{NaCl}$ ) poly $(\mathrm{dA})$ : poly (dT) обладает структурой В-типа с практически эквивалентными конформациями противоположных цепей двойной спирали, т. е. наблюдается согласие между структурой в волокне (кристалле) и структурой в растворе. В отличие от гетерономной модели [5] конформация сахаров в обеих цепях оказывается близкой к $\mathrm{C} 2$ '-endo. В то же время

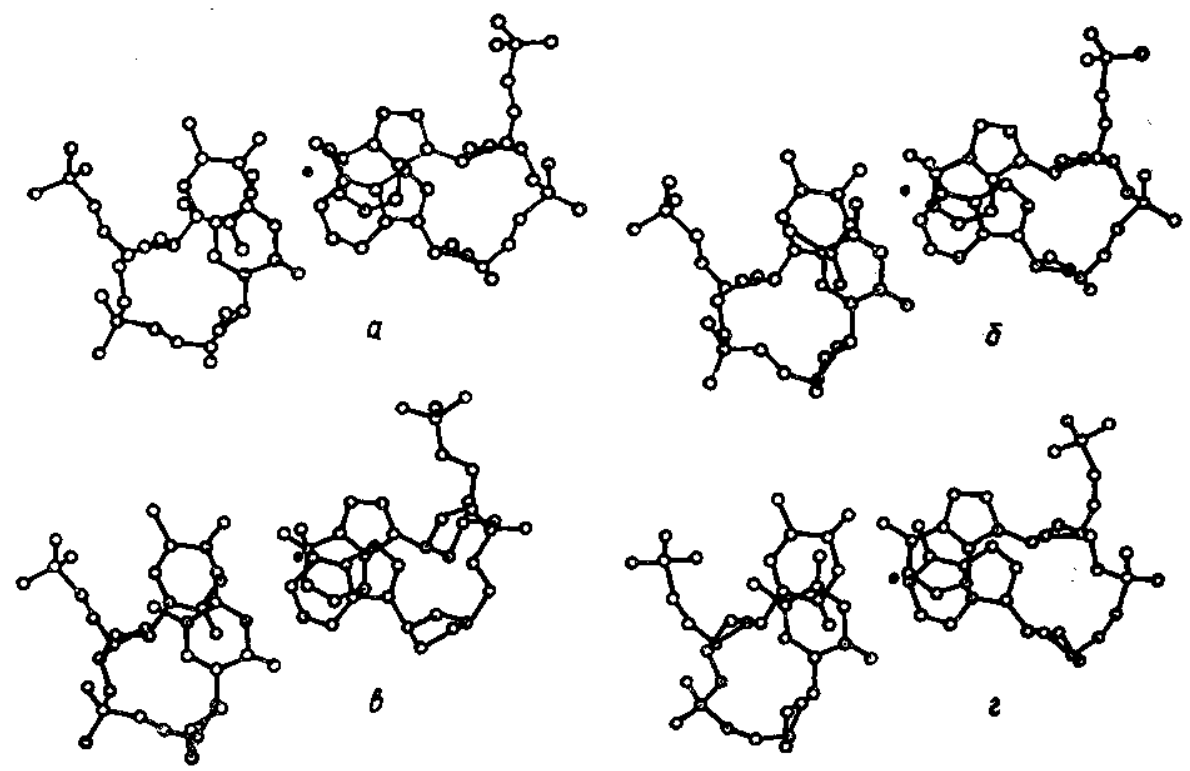

Рис. 3. Проекции вдоль оси спирдли двух последовательных пар нуклеотидов из структур, изображенных на рис. 2 (порядок следования тот же). Отмечены положения осей спиралей.

Fig. 3. Views down the helix axis of two successive pairs of nucleotides from the structures presented in Fig. 2 (the same crder). Helix axis positions are shown.

спектры комбинационного рассеяния (KP) $[7,8]$ указывают на присутствие конформации А-типа, которое становится особенно заметным при понижении температуры до $0-5^{\circ} \mathrm{C}$ и увеличении концентрации соли. Эти данные трудно интерпретировать иначе, чем в рамках модели типа [5]. Однако оказывается, например, что в тех же условиях, когда в спектрах KP [7] еще заметна полоса $816 \mathrm{~cm}^{-1}$, которую относят к C3'-endo конформации, по данным ЯМР [6] все сахара в структype poly $(\mathrm{dA})$ : poly $(\mathrm{dT})$ находятся в $\mathrm{C} 2$ '-endo конформации. С другой стороны, хотя интерпретация спектров резонансного КР, данная в [8], предполагает, что внутрицепочечные взаимодействия между аденинами в двуспиральном poly $(\mathrm{dA})$ : poly $(\mathrm{dT})$ соответствуют А-подобной конформации адениновой цепи, такой подход не кажется убедительным, ибо в конкретных гетерономных и негетерономных сгруктурах все межадениновые стэкинги близки к таковым в В-ДНК (рис. 3 и рис. 5 в [5]).

При всех отмеченных неоднозначностях нельзя, тем не менее, утверждать, что гетерономная структура типа предложенной в работе [5] не может реализоваться в определенных условиях в растворе. Из работы [5] во всяком случае следует, что такая структура стерически приемлема. Разница в энергиях между В-формой и такой, гетерономной, формой в случае poly (dA) : poly (dT) может быть невелика, хотя, как было показано выше (см. Результаты), из рентгеноструктурного эксперимента с волокнами Na-poly $(\mathrm{dA})$ : poly $(\mathrm{dT})$ в действительности следует структура гораздо менее гетерономная, с практически одинаковыми сахаро-фосфатными цепями В-типа. 
Значения конформационных параметров (в градусах) Ca- u Na-poly (dA):poly(dT) (для сравнения приведень параметры гетерономной модели $N$-poly $(d A):$ poly $(d T)$ и модели В-ДНК из работь [5])

The values of the conformational parameters (degrees) of Ca-and $N a-p o l y(d A)$ : : poly $(d T)$. Those of heteronomous model and $B-D N A$ [5] are given for comparison

\begin{tabular}{|c|c|c|c|c|c|c|}
\hline \multirow{2}{*}{ Параметры } & \multirow{2}{*}{$\begin{array}{l}\text { Ca-po- } \\
\text { ly(dA): } \\
\text { :poly(dT) }\end{array}$} & \multicolumn{2}{|c|}{$\mathrm{Na}-\operatorname{poly}\left(\mathrm{d}_{\mathrm{A}}\right): \operatorname{poly}(\mathrm{dT})$} & \multicolumn{2}{|c|}{$\begin{array}{l}\text { Гетерономная } \\
\text { модель [5] }\end{array}$} & \multirow{2}{*}{$\begin{array}{c}\text { В-ДHK } \\
{[5]}\end{array}$} \\
\hline & & $\mathrm{ApA}$ & TpT & $\mathrm{ApA}$ & TpT & \\
\hline $\begin{array}{l}\text { Конформация остова } \\
\mathrm{C} 4^{\prime}-\mathrm{C} 3^{\prime}-\mathrm{O} 3^{\prime}-\mathrm{P} \\
\mathrm{C} 3^{\prime}-\mathrm{O} 3^{\prime}-\mathrm{P}-\mathrm{O} 5^{\prime} \\
\mathrm{O} 3^{\prime}-\mathrm{P}-\mathrm{O} 5^{\prime}-\mathrm{C} 5^{\prime} \\
\mathrm{P}-\mathrm{O} 5^{\prime}-\mathrm{C} 5^{\prime}-\mathrm{C} 4^{\prime} \\
\mathrm{O} 5^{\prime}-\mathrm{C} 5^{\prime}-\mathrm{C} 4^{\prime}-\mathrm{C} 3^{\prime} \\
\mathrm{C} 5^{\prime}-\mathrm{C} 4^{\prime}-\mathrm{C} 3^{\prime}-\mathrm{O} 3^{\prime} \\
\mathrm{O} 1^{\prime}-\mathrm{C} 1^{\prime}-\mathrm{N} 9-\mathrm{C} 4^{\prime} \\
\mathrm{O} 1^{\prime}-\mathrm{C} 1^{\prime}-\mathrm{N} 1-\mathrm{C} 2^{\prime} \\
\text { Конформация сахара } \\
\mathrm{C} 4^{\prime}-\mathrm{O} 1^{\prime}-\mathrm{C} 1^{\prime}-\mathrm{C} 2^{\prime} \\
\mathrm{O} 1^{\prime}-\mathrm{C} 1^{\prime}-\mathrm{C} 2^{\prime}-\mathrm{C} 3^{\prime} \\
\mathrm{C} 1^{\prime}-\mathrm{C} 2^{\prime}-\mathrm{C} 3^{\prime}-\mathrm{C} 4^{\prime} \\
\mathrm{C} 2^{\prime}-\mathrm{C} 3^{\prime}-\mathrm{C} 4^{\prime}-\mathrm{O} 1^{\prime} \\
\mathrm{C} 3^{\prime}-\mathrm{C} 4^{\prime}-\mathrm{O} 1^{\prime}-\mathrm{C} 1^{\prime} \\
\mathrm{Bнутрисахарные} \mathrm{валентные} \mathrm{углы} \\
\mathrm{C} 4^{\prime}-\mathrm{O} 1^{\prime}-\mathrm{C} 1^{\prime} \\
\mathrm{O} 1^{\prime}-\mathrm{C} 1^{\prime}-\mathrm{C} 2^{\prime} \\
\mathrm{C} 1^{\prime}-\mathrm{C} 2^{\prime}-\mathrm{C} 3^{\prime} \\
\mathrm{C} 2^{\prime}-\mathrm{C} 3^{\prime}-\mathrm{C} 4^{\prime} \\
\mathrm{C} 3^{\prime}-\mathrm{C} 4^{\prime}-\mathrm{O} 1^{\prime} \\
\text { Двугранный угол между плоскос- } \\
\text { тями оснований }\end{array}$ & $\begin{array}{r}177 \\
-107 \\
-44 \\
183 \\
40 \\
138 \\
-111 \\
-111\end{array}$ & $\begin{array}{r}178 \\
-107 \\
-44 \\
178 \\
43 \\
133 \\
-110 \\
- \\
-32 \\
39 \\
-30 \\
11 \\
13\end{array}$ & $\begin{array}{r}169 \\
-101 \\
-58 \\
196 \\
46 \\
143 \\
- \\
-113\end{array}$ & $\begin{array}{r}-170 \\
-59 \\
-60 \\
172 \\
54 \\
81 \\
-151 \\
- \\
\\
9 \\
-35 \\
43 \\
-40 \\
21 \\
\\
107 \\
106 \\
103 \\
96 \\
108\end{array}$ & $\begin{array}{r}171 \\
-121 \\
-41 \\
-174 \\
43 \\
152 \\
- \\
-93 \\
-25 \\
42 \\
-42 \\
29 \\
-3\end{array}$ & $\begin{array}{r}-141 \\
-157 \\
-33 \\
138 \\
33 \\
142 \\
-99 \\
--99 \\
-36 \\
46 \\
-38 \\
19 \\
10\end{array}$ \\
\hline
\end{tabular}

Параметры полученных структур Ca- i Na-poly (dA) : poly (dT) хорошо согласуются с данными для кристаллов олигонуклеотидов [14] При общей близости этих структур к классической В-ДНК их прежде всего отличают весьма значительный пропеллер и отрицательный наклон пар оснований (рис. 2). Не исключено, что эти различия могут проявляться на границах между А-Т-последовательностью и случайной последовательностью, например в форме изгибов в ДНК $[15,16]$. В природных ДНК (в том числе в их функциональных участках) встречаются достаточно протяженные (до десятков пар) отрезки $(\mathrm{dA})_{n}:(\mathrm{dT})_{n}$. Их особая структура подтверждается данными ряда работ $[17-19]$. Дополнительная стабилизация структуры poly (dA) : : poly (dT), позволяющая ей сохранять свои особенности в различных условиях, может возникать вследствие особой организации растворителя, важная роль которого отмечалась в работах $[1,20,21]$.

Кальциевая соль poly (dA) : poly (dT) представляет собой первый пример структуры ДНК, детально исследованной в волокнах в виде соли двухвалентного противоиона. Нами была изучена также кальциевая соль природной ДНК, структурные особенности которой повторяют особенности структуры литиевой В-ДНК [12]. Волокна как натриевой [5], так и кальциевой солей poly $(\mathrm{dA})$ : poly $(\mathrm{d} T)$ исследовали при близких относительных влажностях, они отличались только типом противоиона, поэтому причины отличий, которые наблюдаются в их упаковке и структуре, обусловлены только различной организацией их ионно-гидратных оболочек. Дальнейшее выяснение этого вопроса требует более детальных сведений о положении ионов натрия и кальция в указанных структурах. 


\section{STRUCTURE OF THE CALCIUM SALT}

OF POLY (dA) : POLY (dT) AS REVEALED

BY THE X-RAY DIFFRACTION ANALYSIS IN FIBRES

D. G. Alexeev, A. A. Lipanov, I. Ya. Skuratovsky

Institute of Molecular Genetics, Academy of Sciences of the USSR, Moscow

Sum mary

Fibre X-ray diffraction study of the calcium salt of poly $(\mathrm{dA})$ : poly $(\mathrm{dT})$ has resulted in a model of this polynucleotide which is a ten-fold double helix with the pitch of $3.23 \mathrm{n} n$. Antiparallel sugar phosphate chains are fully equivalent in the model, deoxyribose conformation being close to $\mathrm{C} 2^{\prime}$-endo $\left(\delta=138^{\circ}\right)$, and it may be regarded as member of B-family structures. A revised structure of Na-poly $(\mathrm{dA})$ : poly $(\mathrm{dT})$ is suggested, which is also of the B-type and with the highly similar opposite chains.

1. Siructure of the B-DNA cationic shell as revealed by an X-ray diffraction study oi CsDNA / V. N. Bartenev, Eu. I. Golovanov, K. A. Kapitonova et al. // B, Mol.Biol.1983.-169, N 1.- P. 217-234.

2. Локализация ионов $\mathrm{Cs}^{+}$в структуре А-формы ДНК/А. А. Липанов, Д. Г. Алексеев, В. Н. Бартенев и др.// Биофизика.-1986.-31, № 2.-С. 336-338.

3. Скуратовский $И$. Я., Бартенев В. Н. Исследование структуры магниевой и литиевой солей ДНК фага T2 методом дифракции рентгеновских лучей // Молекуляр. биология.-1978.-12, № 6-- С. $1359-1375$.

4. X-ray diffraction and EXAFS studies of the calcium salts of polymeric poly(dA) : : poly(dT) and natural DNA/D. G. Alexeev, L. I. Volkova, I. Ya. Skuratovskii, S. S. Hasnain // Third int. conf. on water and ions in biol. systems: Abstracts.Bucharest $_{n}$ 1984.- P. 205.

5. Heteronomous DNA/S. Arnott, R. Chandrasekaran, 1. H. Hall, L. C. Puigjaner // Nucl. Acids Res.- 1983.-11, N 12.- P. 4141-4155.

6. Sarma M. H., Gupta G., Sarma R. H. Untenability of the heteronomous model for poly (dA) : poly (dT) in solution //J. Biomol. Str. Dyn.-1985.-2, N 6.- P. 1057-1084 .

7. Thomas G. A., Peticolas W. L. Fluctuations in nucleic acids conformations //J. Amer. Chem. Soc.-1983.-105, N 4.- P. 993-996.

8. The poly $(d A)$ strand of poly $(\mathrm{dA})$ : poly $(\mathrm{dT})$ adopts an A-form in solution: UV resonance Raman study / B. Jolles, A. Laigle, L. Chinsky, P. Y. Turpin // Nucl. Acids Res. - 1985.-13, N 6.-P. 2075-2085.

9. Вайнитейн $5 . K$. Дифракция рентгеновских лучей на цепных молекулах.-M. : Наука, 1963- - 371 с.

10. Smith P. J C., Arnott S. LALS: a linked-atoms least-squares resiprocal-space refinement system incorporating stereochemical restrains to supplement sparse diffraction data // Acta Crystallogr.-1978.-A34, N 1.- P. 3-11.

11. Бартенев B. Н., Каменева H. Г., Липанов А. А. Статистические стереохимические модели гибких олигонуклеотидов // Материалы. 6-го симпоз. по конформац. изменениям биополимеров в растворах.- Тбилиси, 1985.- С. 99.

12. The molecular configuration of deoxyribonucleic acid/R. Langridge, D. A. Marvin, W. E. Seeds et al. //J. Mol. Biol. - 1960-2, N 1.-P. 19-64.

13. Hamilton W. C. Significance tests on the crystallographic R factor//Acta crystallogr.-1965.-18, N 4.-P. 502-510.

14. Reversible bending and helix geometry in a B-DNA dodecamer/A. V. Fratini, M. L. Kopka, H. R. Drew, R. E. Dickerson // J. Biol. Chem.-1982.-257, N 24.P. $14686-14707$.

15. Diekmann S., Wang J. C. On the sequence determinants and flexibility of the kinetoplast DNA fragment with abnormal gel electrophoretic mobilities // J. Mol. Biol.1985.-186, N 1.-P. 1-11.

16. Hagerman $P . J$. Sequence-dependent curvature of the helix axis of DNA //Fourth conversation in biomol. stereodyn.: Abstracts.-Albany, 1985.-P. 100-101.

17. Peck $L . J$., Wang $J$. C. Sequence dependence of the helical repeat of DNA in solution // Nature.- I981.--292, N 5821.- P. 375-378.

18. Kunkel G. R., Martinson $H$. G. Nucleosomes will not form on double-stranded RNA or poly $(\mathrm{dA})$ : poly $(\mathrm{d} T)$ tracts in recombinant DNA // Nucl. Acids Res.-- 1981.--9, N 24.- P. 6869-6888.

19. Goodman T. C., Klein R. D., Wells R. D. Effects of neighboring DNA homopolymers on the biochemical and physical properties of the Escherichia coli lactose promoter // J. Biol. Chem. - 1982.-257, N 21.- P. 12970-12978.

20. Drew $H . R$., Dickerson $R$. $E$. Structure of a B-DNA dodecamer. Geometry of hydration // J. Mol. Biol. - 1981.-151, N 3.- P. 535-556.

21. Chuprina V. P. Regularities in formation of the spine of hydration in the DNA minor groove and its influence on the DNA structure // FEBS Letters. - 1985.-186, N 1.- P. $98-102$.

Ин-т молекуляр. генетики АН СССР, Москва

Получено 21.01 .86 